\title{
The Impact of High-Intensity Interval Training on Brain Derived Neurotrophic Factor in Brain: A Mini-Review
}

\author{
Alberto Jiménez-Maldonado1*, Iván Renteria ${ }^{1}$, Patricia C. García-Suárez"1, \\ José Moncada-Jiménez ${ }^{2}$ and Luiz Fernando Freire-Royes ${ }^{3}$ \\ ${ }^{1}$ Facultad de Deportes, Universidad Autónoma de Baja California, Ensenada, Mexico, ${ }^{2}$ Human Movement Sciences \\ Research Center, University of Costa Rica, San José, Costa Rica, ${ }^{3}$ Laboratório de Bioquímica do Exercício, Universidade \\ Federal de Santa Maria, Santa Maria, Brazil
}

\section{OPEN ACCESS}

Edited by:

Adriana Ximenes-da-Silva

Federal University of Alagoas, Brazil

Reviewed by:

Alexander Törpel,

Otto-von-Guericke-Universität

Magdeburg, Germany

Yinghua Yu,

Xuzhou Medical University, China

*Correspondence:

Alberto Jiménez-Maldonado

jimenez.alberto86@uabc.edu.mx

Specialty section:

This article was submitted to Neuroenergetics, Nutrition and Brain

Health,

a section of the journal

Frontiers in Neuroscience

Received: 15 August 2018

Accepted: 29 October 2018

Published: 14 November 2018

Citation:

Jiménez-Maldonado A, Rentería I, García-Suárez PC,

Moncada-Jiménez $J$ and Freire-Royes LF (2018) The Impact of High-Intensity Interval Training on Brain Derived Neurotrophic Factor

in Brain: A Mini-Review.

Front. Neurosci. 12:839.

doi: 10.3389/fnins.2018.00839
The brain-derived neurotrophic factor (BDNF) is a protein mainly synthetized in the neurons. Early evidence showed that BDNF participates in cognitive processes as measured at the hippocampus. This neurotrophin is as a reliable marker of brain function; moreover, recent studies have demonstrated that BDNF participates in physiological processes such as glucose homeostasis and lipid metabolism. The BDNF has been also studied using the exercise paradigm to determine its response to different exercise modalities; therefore, BDNF is considered a new member of the exerciserelated molecules. The high-intensity interval training $(\mathrm{HIIT})$ is an exercise protocol characterized by low work volume performed at a high intensity [i.e., $\geq 80 \%$ of maximal heart rate (HRmax)]. Recent evidence supports the contention that HIIT elicits higher fat oxidation in skeletal muscle than other forms of exercise. Similarly, HIIT is a good stimulus to increase maximal oxygen uptake $\left(\mathrm{VO}_{2} \mathrm{max}\right)$. Few studies have investigated the impact of HIIT on the BDNF response. The present work summarizes the effects of acute and long-term HIIT on BDNF.

Keywords: brain-derived neurotrophic factor, high-intensity interval training, brain, health, physical exercise

\section{INTRODUCTION}

Physical exercise (PE) is considered a subcategory of the physical activity domain (Caspersen et al., 1985; Physical Activity Guidelines Advisory Committee, 2008). As opposed to physical activity, PE is characterized for being planned, structured, and repetitive, with the inherent goal of improving one or more components of the physical fitness, physical performance, or health (Caspersen et al., 1985; Physical Activity Guidelines Advisory Committee , 2008). The PE prescription is usually reported as exercise mode, intensity, frequency, and duration of the activity.

Several training modalities have been developed over the years with the aim of improving cardiorespiratory fitness, musculoskeletal function, and metabolic activity. Among these, aerobic, endurance, and/or resistance (i.e., strength exercise) are the most common exercise training modalities (Kang and Ratamess, 2014). The aerobic exercise (AE) is also known as moderateintensity continuous training (MICT), and it is usually performed over long periods of time (e.g., $\geq 30$-min to moderate intensity, performing exercises such as walking, cycling, jogging, and swimming) (Garber et al., 2011). Although the benefits of MICT on health related parameters in humans are well studied (Aldred et al., 1995; Poehlman et al., 2000; Mador et al., 2004; 
Frøsig et al., 2007; Camargo et al., 2008; Bell et al., 2010; Fisher et al., 2015; Daabis et al., 2017); currently, the world population considers that lack of time as the main barrier to practice $\mathrm{AE}$ regularly (Weston et al., 2014; Fisher et al., 2015).

Scientists and exercise professionals have focused on studying the impact of short exercise bouts on human physiology with the aim of optimizing time use (Gibala et al., 2006); for instance, high-intensity interval training (HIIT). The HIIT refers to exercise characterized by relatively short bursts of vigorous activity, interspersed by rest or low-intensity recovery exercise. In general, HIIT is performed on a training session lasting $\leq 30$-min, including warm-up and cool down stages (Gibala and Jones, 2013; Gillen and Gibala, 2013; Weston et al., 2014). The high-intensity bouts should be performed at near maximal effort, reaching intensities between 80 and $100 \%$ of the maximal heart rate (HRmax) (Gibala et al., 2014; Saanijoki et al., 2018). The exertion is performed no longer than 60-s (Gillen and Gibala, 2013), and the recovery periods (low-intensity exercise or rest) can be up to 4-min (Burgomaster et al., 2005, 2006; Gibala and McGee, 2008). Besides Gibala's protocols, others have reported different high- and low-exercise bout durations (Saucedo Marquez et al., 2015; Lira et al., 2017; Stöggl and Björklund, 2017); however, the training session has been kept within $\leq 30$ - $\min$.

In addition, HIIT can be performed on cyclical exercises such as bicycling (Saucedo Marquez et al., 2015), running (Lira et al., 2017), swimming (Courtright et al., 2016), and wholebody exercise (Machado et al., 2017; Schleppenbach et al., 2017). Several physiological adaptations of HIIT have been reported to improve physical performance in humans (Burgomaster et al., 2005, 2006; Gibala et al., 2006; Talanian et al., 2007; Connolly et al., 2017). The effects of HIIT on brain function have been also reported; however, there available evidence is scarce (Afzalpour et al., 2015; Lucas et al., 2015; Coetsee and Terblanche, 2017; Santos-Concejero et al., 2017; So et al., 2017; Freitas et al., 2018; Robinson et al., 2018). Indeed, the current evidence showed a positive impact of HIIT in brain, specifically in neurotrophin expression and function. In this context, the aim of this work is to briefly describe the current knowledge regarding the acute and long-term effects of HIIT on brain-derived neurotrophic factor (BDNF) in brain. It is known that BDNF is a protein that plays a key role to maintain or improve several brain functions (Vaynman et al., 2003, 2004; Duman and Monteggia, 2006; Duman and Li, 2012; Fernandes et al., 2017).

\section{HIGH-INTENSITY INTERVAL TRAINING (HIIT): AN EFFICIENT TOOL TO IMPROVE PHYSICAL PERFORMANCE, METABOLISM, AND BRAIN FUNCTION}

As described, HIIT refers to exercise characterized by relatively short bursts of vigorous activity, interspersed by rest or lowintensity recovery exercise (Gibala and Jones, 2013; Gillen and Gibala, 2013; Weston et al., 2014). Previous scientific reports have indicated that HIIT is perceived as an exercise modality eliciting higher exhaustion compared to MICT (Saanijoki et al., 2015, 2018). However, HIIT is considered more enjoyable than MICT (Heisz et al., 2016); in agreement with this, HIIT has been proposed as an excellent strategy aimed to increase adherence to exercise programs in sedentary people (Heisz et al., 2016). In this section, the impact of HIIT on the human physiology (physical performance, metabolism, and brain function) will be briefly described. In sport, scientific reports indicate that HIIT was popularized by the runner Emil Zatopek around 1950 [see Billat's (2001) review on the historical analysis of HIIT]; in fact, several coaches think that HIIT played a key role on Zatopek's successful sport career. Similarly, recent evidence indicates that HIIT improves physical performance (e.g., speed, agility) in team sport athletes such as soccer and basketball (Iaia et al., 2015; Sanchez-Sanchez et al., 2018).

Regarding to metabolic dysfunctions in glucose and lipids induced by sedentary lifestyle and hypercaloric diets, several evidence showed that HIIT is an efficient stimulus to improve lipid and glucose metabolism. Concretely, Talanian et al. (2007), reported that seven sessions of HIIT increased fat oxidation in skeletal muscle in recreationally active women. Similarly, others demonstrated that HIIT interventions enhance insulin sensitivity, glucose control, and cardiorespiratory fitness in sedentary women (Connolly et al., 2016, 2017). In addition to research on women, others reported that HIIT increases muscle oxidative capacity in recreationally active men (Burgomaster et al., 2005, 2006; Gibala et al., 2006).

The central nervous system (CNS) response to HIIT has been reported in spinal cord (Astorino and Thum, 2018) and brain studies (Coetsee and Terblanche, 2017; SantosConcejero et al., 2017; Robinson et al., 2018). For instance, a 16-week HIIT program elicited higher oxygen utilization and cerebral oxygenation than MICT in older people (Coetsee and Terblanche, 2017); similar results were found in younger adults (Robinson et al., 2018). In these studies, the BDNF's response was dependent of the exercise intensity. However, the molecular mechanisms explaining these brain adaptations to HIIT are yet to be elucidated.

\section{BRAIN-DERIVED NEUROTROPHIC FACTOR (BDNF): A PROTEIN SENSITIVE TO EXERCISE IN BRAIN}

\section{BDNF Function in Brain and Periphery}

The BDNF is a protein member of the neurotrophin family, and it is found in the nervous system and peripheral organs such as skeletal muscle (Funakoshi et al., 1993; Conner et al., 1997; Matthews et al., 2009). In the CNS, the neurons are the principal source of BDNF (Mowla et al., 2001), and evidence suggests that BDNF plays a key role in memory and learning processes (Erickson et al., 2011). Moreover, molecular evidence indicates that this neurotrophin through a tyrosine kinase $\mathrm{b}$ receptor (TrkB) increases long-term potentiation, neurogenesis, axonal growth, and synaptogenesis (Tyler and Pozzo-Miller, 2001; Vaynman et al., 2003, 2004; Fernandes et al., 2017). Besides 


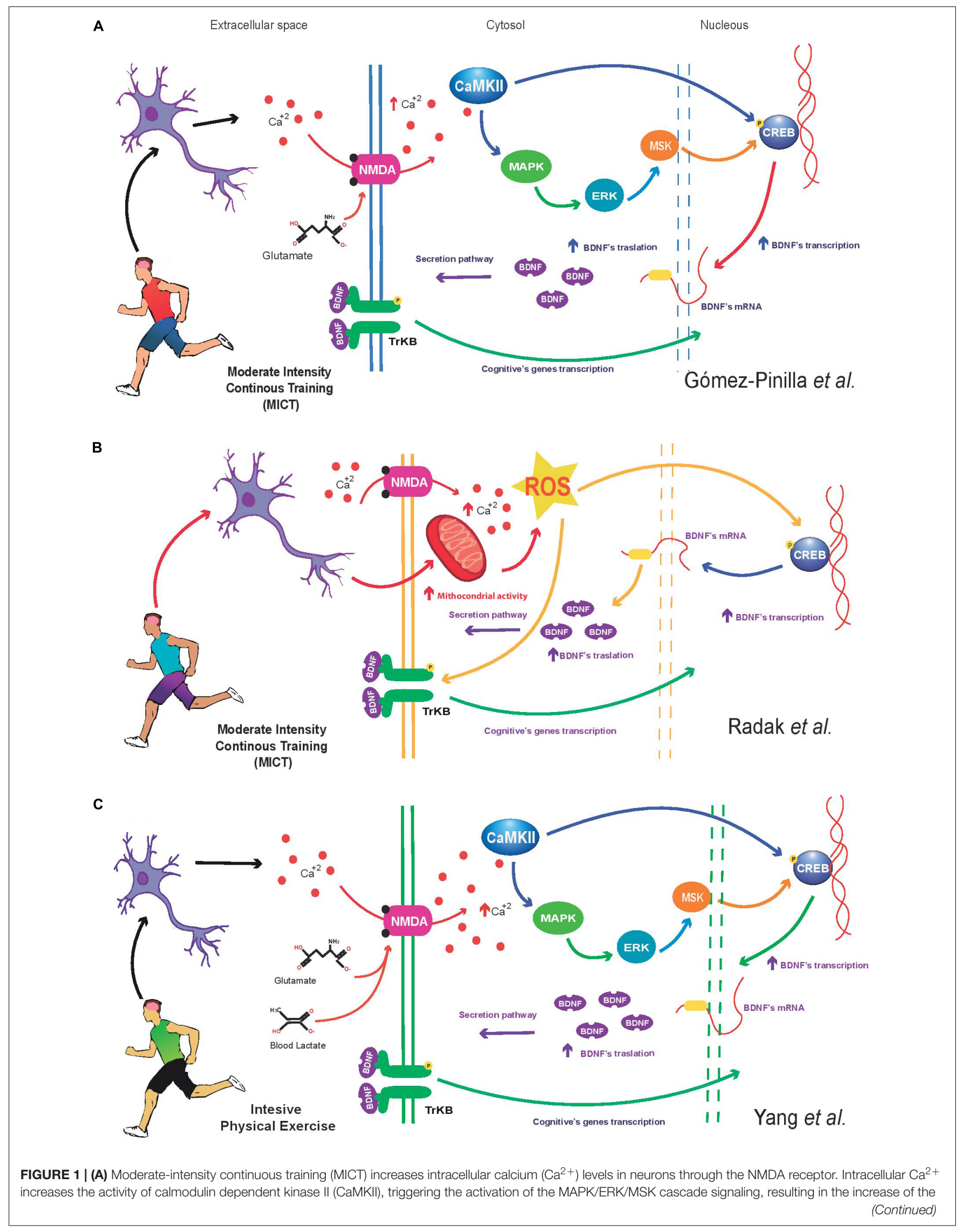




\section{FIGURE 1 | Continued}

expression and phosphorylation of CAMP response element-binding protein (CREB). Finally, CREB enhance the Bdnf transcription. This molecular mechanism described above result in a higher BDNF protein, the neurotrophin is released by the neuron to induce transcription of cognitive genes. The present model is based on Gomez-Pinilla's studies (Fernandes et al., 2017). (B) MICT enhances the mitochondrial activity in neurons. Higher mitochondrial activity increases reactive oxygen species (ROS) from complexes I and III. The change in ROS levels modify and regulate a wide of signaling process including the CREB-BDNF signaling pathway. Once activated, BDNF regulates a positive feedback mechanism to induce the cognitive genes transcription. Additionally, the aerobic exercise increases the calcium concentration in neurons; this ion through the calpain and xanthine oxidase increases the ROS that consequently increase the CREB's activation and Bdnf expression. (C) Exercise performed at high intensity ( $\geq 80 \% \mathrm{HRmax}$ ) activates several metabolic pathways in muscle (including glycolysis), this condition generates a higher systemic blood lactate concentration reaching the brain, this metabolite can be oxidized by astrocytes or neurons to produce glucose (Dienel and Hertz, 2001). In addition, experimental evidence indicates that lactate increase NMDA activity and intracellular $\mathrm{Ca}^{2+}$ levels in neurons. Indeed, it is possible that the lactate in neurons enhance the CaMKII activity and the MAPK/ERK/MSK signaling to induce the CREB's activation and Bdnf expression. Finally, the BDNF activate a positive loop to induce the expression of cognitive genes (Yang J. et al., 2014).

the local effect of BDNF in the brain, some authors suggest that the brain is the major source of circulating BDNF at rest and during exercise (Rasmussen et al., 2009; Seifert et al., 2010). In the periphery, studies performed in rodent and human tissues have revealed that BDNF regulates other physiological pathways such as glucose metabolism (Hanyu et al., 2003; Jiménez-Maldonado et al., 2014), and fat oxidation (Matthews et al., 2009).

\section{Molecular Mechanism Induced by Physical Exercise Increasing Brain BDNF}

Several stimuli can increase BDNF's expression and function. In rodents, the kainic acid exposure increased hippocampal BDNF (protein) levels (Rudge et al., 1998), resulting from an enhancement in glutamatergic signaling. Other evidence suggests that intermittent hypoxia increases BDNF levels in neurons of the primary motor cortex (Satriotomo et al., 2016). In addition to these findings, it is widely known that $\mathrm{PE}$ is an effective stimulus to increase BDNF synthesis in the brain (Oliff et al., 1998; Vaynman et al., 2003, 2004; Erickson et al., 2011), and the periphery (Dinoff et al., 2016, 2017).

Regarding to the impact of the PE on increasing BDNF in brain, different molecular mechanisms have been proposed to explain how PE (mainly MICT) enhances BDNF synthesis in neurons. The Gomez-Pinilla's group suggests that PE increases the intracellular $\mathrm{Ca}^{2+}$ levels in neuronal cells (Fernandes et al., 2017). This ion activates CaMKII indirectly; and once active, this kinase increases the MAP-K pathway to phosphorylate CRE-binding protein and activate the CREB transcription, and consequently Bdnf transcription (Vaynman et al., 2004; Fernandes et al., 2017; Figure 1A). Another model suggests that PE induces BDNF synthesis in the brain by enhancing the activity of reactive oxygen species (ROS) (Radak et al., 2016). Based on Radak et al's. proposal, PE increases the mitochondrial activity in neurons; and it is known that higher mitochondrial activity produce excessive ROS. Thus, ROS enhance the activity of CREbinding protein, to activate the CREB and $B d n f$ transcription (Radak et al., 2016; Figure 1B). Additionally, the Radak's model indicates that the exercise increases the $\mathrm{Ca}^{2+}$ in neurons; this ion through the calpain and xanthine oxidase induces higher ROS production in brain as well (Takuma et al., 1999; Kahlert et al., 2005). In addition to the previous mechanism, it has been suggested that systemic molecules such as the lactate synthesized during intensive $\mathrm{PE} \mathrm{(} \geq 80 \% \mathrm{HRmax}$ ) can activate the BDNF production (Bergersen, 2015). However, this molecular mechanism of BDNF production is still poorly understood. Experimental evidence has shown higher NMDA receptor activity in the presence of lactate; furthermore, high lactate concentrations are associated to increased neuronal $\mathrm{Ca}^{2+}$ levels (Yang J. et al., 2014) and higher Bdnf transcription (Yang J.L. et al., 2014). It is likely that lactate synthesized during PE reach the neurons and increase the NMDA receptor activity to increase the $\mathrm{Ca}^{2+}$ concentration in neurons, and $\mathrm{Ca}^{2+}$ activates CaMKII, and consequently, the kinase phosphorylates activating the MAPK/ERK signaling pathway to enhance $B d n f$ transcription (Figure 1C).

\section{THE EFFECTS OF HIIT ON BDNF}

\section{Animal Models}

As previously described, HIIT is characterized by exercise bouts of high-intensity and low volume. Regarding exercise intensity, evidence in healthy rodents has shown that brain BDNF synthesis was higher in animals performing a high-intensity training compared to those animals performing a low-intensity training and sedentary rodents (de Almeida et al., 2013). However, the authors used a continuous training protocol; the training time was similar in both models (low- and high-intensity training, 30-min/session), which means that the HIIT characteristics were unattained.

There is evidence regarding the long-term effects of HIIT on BDNF synthesis in rodents (Afzalpour et al., 2015; So et al., 2017; Freitas et al., 2018). Thirty HIIT sessions significantly increased BDNF levels (protein) in the brain compared with continuous training protocol and a control group (Afzalpour et al., 2015). The authors discussed that HIIT increased hydrogen peroxide $\left(\mathrm{H}_{2} \mathrm{O}_{2}\right)$ and Tumor Necrosis Factor Alpha (TNF$\alpha$ ) concentration in brain; and these molecules could activate the BDNF synthesis (Wang et al., 2006; Bałkowiec-Iskra et al., 2011) or CREB (Pugazhenthi et al., 2003), a transcription factor positively regulating BDNF synthesis. However, although the previous paper found a positive effect of HIIT on BDNF, the authors did not report a specific anatomical region sensitive to elevations on the neurotrophin following HIIT. Consequently, others evaluated with more detail the impact of HIIT on BDNF in the hippocampus (Freitas et al., 2018). In the study by Freitas et al. (2018), 36 sessions of HIIT elevated BDNF levels in the hippocampal region of healthy rats. However, the molecular 


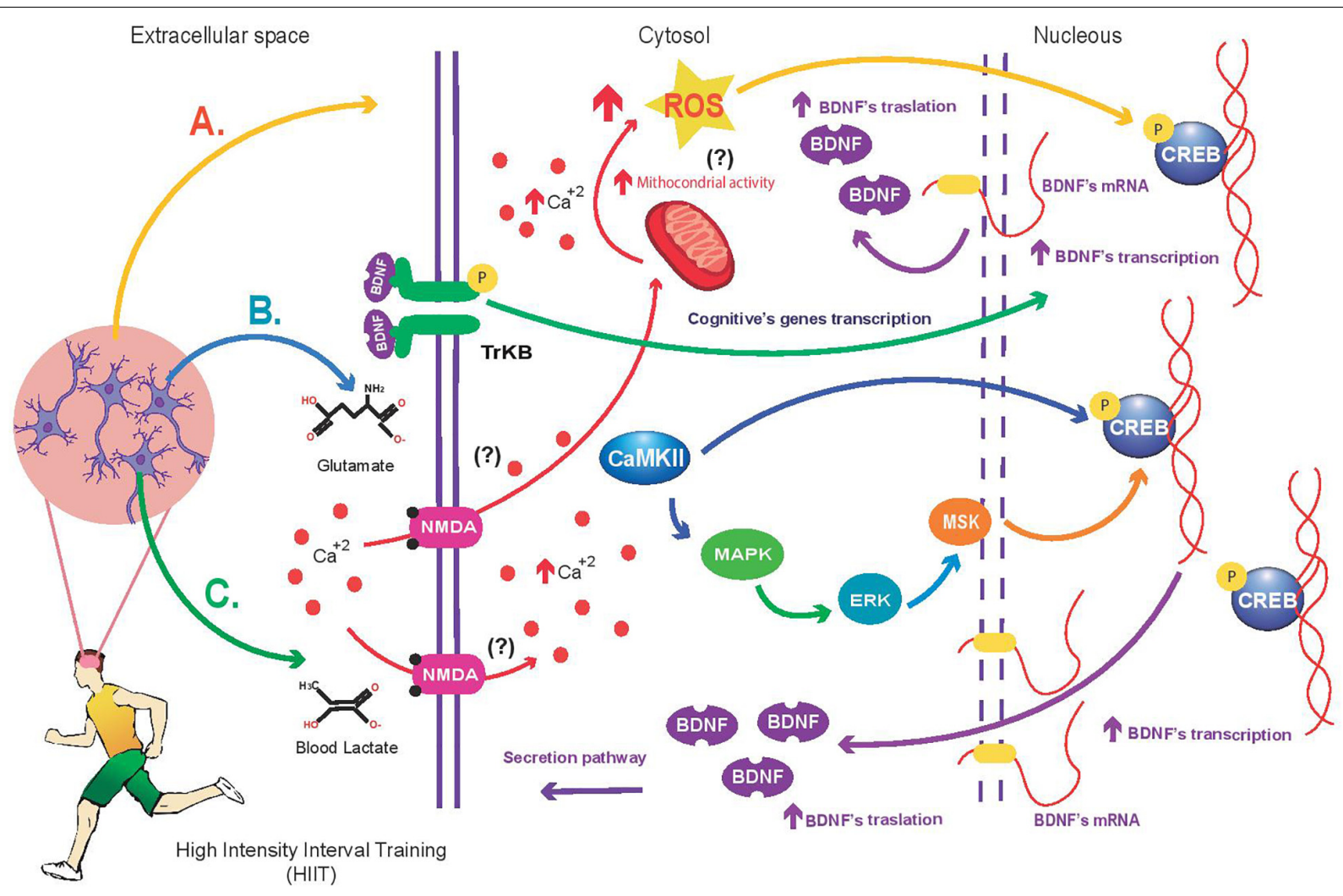

FIGURE 2 | Mechanism proposed about the High Intensity Interval Training (HIIT) impact on BDNF synthesis in brain. (A) HIIT increases mitochondrial activity (not reported) and ROS concentration in neurons (Afzalpour et al., 2015) compared with MICT. ROS induce higher Creb-Bdnf transcription and signaling than MICT (not reported). (B) HIIT causes greater $\mathrm{Ca}^{2+}$ concentration in neurons than MICT (not reported); this condition enhances the CaMKIl activity and MAPK/ERK/MSK signaling to activate the Creb-Bdnf transcription and neuronal plasticity. Additionally, the intracellular calcium can increase the ROS generation in neurons. Once synthetized, ROS can activate Creb-Bdnf transcription. Currently, there is not experimental evidence to indicate that HIIT triggers more this mechanism than MICT. (C) HIIT elevate systemic blood lactate concentration, and consequently enhance the NMDA receptor activity to increase intracellular $\mathrm{Ca}^{2+}$ concentration in neurons (not reported). The ion activates the CaMKIl activity and MAPK/ERK/MSK signaling to induce the Creb-Bdnf transcription and neuronal plasticity (not reported). (?): not reported.

mechanism responsible for increasing BDNF synthesis was not demonstrated in the study. In agreement with their results, the authors suggested that 36 HIIT sessions increased BDNF levels and attenuated hippocampal oxidative damage (Freitas et al., 2018).

\section{Human Models}

There are reports on the effect of a single HIIT session on BDNF (Saucedo Marquez et al., 2015; Cabral-Santos et al., 2016; Slusher et al., 2018). For instance, a single session of supramaximal HIIT elevated serum BDNF levels (Slusher et al., 2018), suggesting increases in BDNF secretion of the platelets unrelated to brain secretion (Slusher et al., 2018). Saucedo Marquez et al. (2015), found that HIIT was a more powerful stimulus to elevate systemic (serum) BDNF compared to MICT. The exercise modality employed in their study (cycle-ergometer) did not induce muscle damage (Saucedo Marquez et al., 2015). Therefore, the higher BDNF levels were not caused by platelet activation to increase the BDNF secretion (Saucedo Marquez et al., 2015), suggesting that PE itself is enough stimuli that lead to higher circulating BDNF levels. Thus, the higher serum BDNF levels following HIIT resulted from a greater synthesis of BDNF in the brain. The authors discussed that a single bout of HIT induced higher brain $\mathrm{H}_{2} \mathrm{O}_{2}$ and TNF- $\alpha$ levels. These molecules activate the signaling of peroxisome proliferator-activated receptor- $\gamma$ coactivator (PGC$1 \alpha)$ to enhance neuron BDNF synthesis (Saucedo Marquez et al., 2015). Similarly, a single session of HIIT significantly increased peripheral plasmatic BDNF levels immediately following the exercise (Cabral-Santos et al., 2016). However, after 60-min that the HIIIT session ended, BDNF concentrations returned to baseline levels (Cabral-Santos et al., 2016). Regarding that plasmatic BDNF levels reflect the BDNF secretion from the brain (Lommatzsch et al., 2005); the Cabral-Santos data reflect the HIIT impact on BDNF in brain. The authors suggested that brain hypoxia induced by HIIT was the main factor explaining their results (Cabral-Santos et al., 2016).

Finally, the long-term effects of HIIT on systemic (serum) BDNF levels have been also reported (MurawskaCialowicz et al., 2015). In the study, participants performed whole-body exercises for 3 months, and the protocol was effective at increasing serum BDNF concentrations. However, the BDNF source was not elucidated. 


\section{PERSPECTIVES AND CONCLUDING REMARKS}

Studies performed in rodents (Tsuchida et al., 2001; Hanyu et al., 2003; Yamanaka et al., 2006; Jiménez-Maldonado et al., 2014) and humans (Bulloì et al., 2007; Krabbe et al., 2007; Li et al., 2016) have demonstrated that BDNF participates in glucose and lipid metabolism (Matthews et al., 2009). Therefore, this molecule also is known as metabotrophin (Gomez-Pinilla et al., 2008). Several health conditions such as type 2 diabetes, obesity, metabolic syndrome, and cardiovascular diseases are mainly caused by dysfunctional metabolic mechanisms and sedentary lifestyle. Therefore, it is important to identify efficient stimuli to increase the BDNF function in population with high risk to suffer metabolic diseases or in people who are suffering metabolic diseases. Thus, HIIT seems be a good stimulus to enhance the BDNF action. However, the impact of HIIT on BDNF and its effect on glucose and lipid metabolism is poorly studied. Further experimental studies are necessary to elucidate the impact of HIIT on BDNF and its effect on glucose and lipid metabolism in humans with metabolic or cardiovascular diseases. In addition, during modern-life diseases (Type II diabetes, obesity, and metabolic syndrome); the brain function is also affected (Cisternas et al., 2015; Agrawal et al., 2016). Therefore, studies are needed to assess the impact of HIIT interventions on BDNF synthesis and signaling pathways in brain under morbid conditions. The current work proposes a model about the impact of HIIT on BDNF expression in the brain (Figure 2). It will be reasonable to use previous HIIT protocols that reported a positive impact in peripheral BDNF when thinking about the design of HIIT protocols aimed at increasing BDNF synthesis and brain

\section{REFERENCES}

Afzalpour, M. E., Chadorneshin, H. T., Foadoddini, M., and Eivari, H. A. (2015). Comparing interval and continuous exercise training regimens on neurotrophic factors in rat brain. Physiol. Behav. 147, 78-83. doi: 10.1016/j.physbeh.2015. 04.012

Agrawal, R., Noble, E., Vergnes, L., Ying, Z., Reue, K., and Gomez-Pinilla, F. (2016). Dietary fructose aggravates the pathobiology of traumatic brain injury by influencing energy homeostasis and plasticity. J. Cereb. Blood Flow Metab. 36, 941-953. doi: 10.1177/0271678x15606719

Aldred, H. E., Hardman, A. E., and Taylor, S. (1995). Influence of 12 weeks of training by brisk walking on postprandial lipemia and insulinemia in sedentary middle-aged women. Metabolism 44, 390-397. doi: 10.1016/0026-0495(95) 90172-8

Astorino, T. A., and Thum, J. S. (2018). Interval training elicits higher enjoyment versus moderate exercise in persons with spinal cord injury. J. Spinal Cord Med. 41, 77-84. doi: 10.1080/10790268.2016.1235754

Bałkowiec-Iskra, E., Vermehren-Schmaedick, A., and Balkowiec, A. (2011). Tumor necrosis factor-alpha increases brain-derived neurotrophic factor expression in trigeminal ganglion neurons in an activity-dependent manner. Neuroscience 180, 322-333. doi: 10.1016/j.neuroscience.2011.02.028

Bell, G. J., Harber, V., Murray, T., Courneya, K. S., and Rodgers, W. (2010). A comparison of fitness training to a pedometer-based walking program matched for total energy cost. J. Phys. Act Health 7, 203-213. doi: 10.1123/jpah. 7.2.203

Bergersen, L. H. (2015). Lactate transport and signaling in the brain: potential therapeutic targets and roles in body-Brain interaction. J. Cereb. Blood Flow Metab. 35, 176-185. doi: 10.1038/jcbfm.2014.206 signaling. For example, sprint interval training (60-s run at $100 \%$ $\mathrm{VO}_{2}$ max, interspersed with 60-s passive recovery) (Cabral-Santos et al., 2016). In addition, the peak power output (PPO-Watts-) can also be used to design a HIIT protocol (Saucedo Marquez et al., 2015); for instance, the protocol could consist in pedaling for 60 -s at $90 \%$ of PPO, alternating with 60 -s of active rest at 60 Watts (total duration of HIIT is 20-min) (Saucedo Marquez et al., 2015). Finally, a recent report performed in overweight subjects showed that a HIIT protocol designed using heart rate as the main variable to establish the workload intensities is not a good stimulus to increase the peripheral BDNF (DomínguezSanchéz et al., 2018). Further studies are needed to determine whether heart rate may be considered as a reliable physiological variable used to design a HIIT protocol aimed at increasing circulating BDNF in non-obese subjects.

\section{AUTHOR CONTRIBUTIONS}

AJ-M conceived the review focus, reviewed the literature, wrote the first draft, and finalized the manuscript. IR and PG-S reviewed the literature, wrote the first draft, and finalized the manuscript. JM-J and LF-R finalized the manuscript. All authors approved the final version of the manuscript.

\section{FUNDING}

This work was partially supported by the funding Apoyo a la Incorporación de NPTC PRODEP 2017 No. UABC-PTC 660 (to AJ-M).

Billat, L. V. (2001). Interval training for performance: a scientific and empirical practice. Special recommendations for middle- and long-distance running. Part I: aerobic interval training. Sports Med. 31, 13-31. doi: 10.2165/00007256200131010-00002

Bulloì, M., Peeraully, M. R., Trayhurn, P., Folch, J., and Salas-Salvadoì, J. (2007). Circulating nerve growth factor levels in relation to obesity and the metabolic syndrome in women. Eur. J. Endocrinol. 157, 303-310. doi: 10.1530/eje-06-0716

Burgomaster, K. A., Heigenhauser, G. J., and Gibala, M. J. (2006). Effect of short-term sprint interval training on human skeletal muscle carbohydrate metabolism during exercise and time-trial performance. J. Appl. Physiol. 100, 2041-2047. doi: 10.1152/japplphysiol.01220.2005

Burgomaster, K. A., Hughes, S. C., Heigenhauser, G. J., Bradwell, S. N., and Gibala, M. J. (2005). Six sessions of sprint interval training increases muscle oxidative potential and cycle endurance capacity in humans. J. Appl. Physiol. 98, 1985-1990. doi: 10.1152/japplphysiol.01095.2004

Cabral-Santos, C., Castrillon, C. I., Miranda, R. A., Monteiro, P. A., Inoue, D. S., Campos, E. Z., et al. (2016). Inflammatory cytokines and BDNF response to high-intensity intermittent exercise: effect the exercise volume. Front. Physiol. 7:509. doi: 10.3389/fphys.2016.00509

Camargo, M. D., Stein, R., Ribeiro, J. P., Schvartzman, P. R., Rizzatti, M. O., and Schaan, B. D. (2008). Circuit weight training and cardiac morphology: a trial with magnetic resonance imaging. Br. J. Sports Med. 42, 141-145; discussion 145. doi: 10.1136/bjsm.2007.038281

Caspersen, C. J., Powell, K. E., and Christenson, G. M. (1985). Physical activity, exercise, and physical fitness: definitions and distinctions for health-related research. Public Health Rep. 100, 126-131.

Cisternas, P., Salazar, P., Serrano, F. G., Montecinos-Oliva, C., Arredondo, S. B., Varela-Nallar, L., et al. (2015). Fructose consumption reduces hippocampal 
synaptic plasticity underlying cognitive performance. Biochim. Biophys. Acta Mol. Basis Dis. 1852, 2379-2390. doi: 10.1016/j.bbadis.2015.08.016

Coetsee, C., and Terblanche, E. (2017). Cerebral oxygenation during cortical activation: the differential influence of three exercise training modalities. A randomized controlled trial. Eur. J. Appl. Physiol. 117, 1617-1627. doi: 10. 1007/s00421-017-3651-8

Conner, J. M., Lauterborn, J. C., Yan, Q., Gall, C. M., and Varon, S. (1997). Distribution of brain-derived neurotrophic factor (BDNF) protein and mRNA in the normal adult rat CNS: evidence for anterograde axonal transport. J. Neurosci. 17, 2295-2313. doi: 10.1523/JNEUROSCI.17-07-02295. 1997

Connolly, L. J., Bailey, S. J., Krustrup, P., Fulford, J., Smietanka, C., and Jones, A. M. (2017). Effects of self-paced interval and continuous training on health markers in women. Eur. J. Appl. Physiol. 117, 2281-2293. doi: 10.1007/s00421-0173715-9

Connolly, L. J., Nordsborg, N. B., Nyberg, M., Weihe, P., Krustrup, P., and Mohr, M. (2016). Low-volume high-intensity swim training is superior to highvolume low-intensity training in relation to insulin sensitivity and glucose control in inactive middle-aged women. Eur. J. Appl. Physiol. 116, 1889-1897. doi: 10.1007/s00421-016-3441-8

Courtright, S. P., Williams, J. L., Clark, I. E., Pettitt, R. W., and Dicks, N. D. (2016). Monitoring interval-training responses for swimming using the 3-min all-out exercise test. Int. J. Exerc. Sci. 9, 545-553.

Daabis, R., Hassan, M., and Zidan, M. (2017). Endurance and strength training in pulmonary rehabilitation for COPD patients. Egpt. J. Chest Dis. Tuberc. 66, 231-236. doi: 10.1016/j.ejcdt.2016.07.003

de Almeida, A. A., Gomes, da Silva, S., Fernandes, J., Peixinho-Pena, L. F., Scorza, F. A., et al. (2013). Differential effects of exercise intensities in hippocampal BDNF, inflammatory cytokines and cell proliferation in rats during the postnatal brain development. Neurosci. Lett. 553, 1-6. doi: 10.1016/j.neulet. 2013.08.015

Dienel, G. A., and Hertz, L. (2001). Glucose and lactate metabolism during brain activation. J. Neurosci. Res. 66, 824-838. doi: 10.1002/jnr.10079

Dinoff, A., Herrmann, N., Swardfager, W., and Lanctôt, K. L. (2017). The effect of acute exercise on blood concentrations of brain-derived neurotrophic factor in healthy adults: a meta-analysis. Eur. J. Neurosci. 46, 1635-1646. doi: 10.1111/ ejn. 13603

Dinoff, A., Herrmann, N., Swardfager, W., Liu, C. S., Sherman, C., Chan, S., et al. (2016). The effect of exercise training on resting concentrations of peripheral brain-derived neurotrophic factor (BDNF): a meta-analysis. PLoS One 11:e0163037. doi: 10.1371/journal.pone.0163037

Domínguez-Sanchéz, M. A., Bustos-Cruz, R. H., Velasco-Orjuela, G. P., Quintero, A. P., Tordecilla-Sanders, A., Correa-Bautista, J. E., et al. (2018). Acute effects of high intensity, resistance, or combined protocol on the increase of level of neurotrophic factors in physically inactive overweight adults: the brainfit study. Front. Physiol. 9:741. doi: 10.3389/fphys.2018.00741

Duman, R. S., and Li, N. (2012). A neurotrophic hypothesis of depression: role of synaptogenesis in the actions of NMDA receptor antagonists. Philos. Trans. $R$. Soc. Lond. B Biol. Sci. 367, 2475-2484. doi: 10.1098/rstb.2011.0357

Duman, R. S., and Monteggia, L. M. (2006). A neurotrophic model for stressrelated mood disorders. Biol. Psychiatry 59, 1116-1127. doi: 10.1016/j.biopsych. 2006.02.013

Erickson, K. I., Voss, M. W., Prakash, R. S., Basak, C., Szabo, A., Chaddock, L., et al. (2011). Exercise training increases size of hippocampus and improves memory. Proc. Natl. Acad. Sci. U.S.A. 108, 3017-3022. doi: 10.1073/pnas.10159 50108

Fernandes, J., Arida, R. M., and Gomez-Pinilla, F. (2017). Physical exercise as an epigenetic modulator of brain plasticity and cognition. Neurosci. Biobehav. Rev. 80, 443-456. doi: 10.1016/j.neubiorev.2017.06.012

Fisher, G., Brown, A. W., Bohan Brown, M. M., Alcorn, A., Noles, C., Winwood, L., et al. (2015). High intensity interval- vs moderate intensity- training for improving cardiometabolic health in overweight or obese males: a randomized controlled trial. PLoS One 10:e138853. doi: 10.1371/journal.pone.013 8853

Freitas, D. A., Rocha-Vieira, E., Soares, B. A., Nonato, L. F., Fonseca, S. R., Martins, J. B., et al. (2018). High intensity interval training modulates hippocampal oxidative stress, BDNF and inflammatory mediators in rats. Physiol. Behav. 184, 6-11. doi: 10.1016/j.physbeh.2017.10.027
Frøsig, C., Rose, A. J., Treebak, J. T., Kiens, B., Richter, E. A., and Wojtaszewski, J. F. (2007). Effects of endurance exercise training on insulin signaling in human skeletal muscle: interactions at the level of phosphatidylinositol 3-kinase, Akt, and AS160. Diabetes Metab. Res. Rev. 56, 2093-2102. doi: 10.2337/db061698

Funakoshi, H., Frisen, J., Barbany, G., Timmusk, T., Zachrisson, O., Verge, V. M., et al. (1993). Differential expression of mRNAs for neurotrophins and their receptors after axotomy of the sciatic nerve. J. Cell Biol. 123, 455-465. doi: 10.1083/jcb.123.2.455

Garber, C. E., Blissmer, B., Deschenes, M. R., Franklin, B. A., Lamonte, M. J., Lee, I.-M., et al. (2011). Quantity and quality of exercise for developing and maintaining cardiorespiratory, musculoskeletal, and neuromotor fitness in apparently healthy adults: guidance for prescribing exercise. Med. Sci. Sports Exerc. 43, 1334-1359. doi: 10.1249/MSS.0b013e318213fefb

Gibala, M. J., Gillen, J. B., and Percival, M. E. (2014). Physiological and healthrelated adaptations to low-volume interval training: influences of nutrition and sex. Sports Med. 44, 127-137. doi: 10.1007/s40279-014-0259-6

Gibala, M. J., and Jones, A. M. (2013). Physiological and performance adaptations to high-intensity interval training. Nestle Nutr. Inst. Workshop Ser. 76, 51-60. doi: 10.1159/000350256

Gibala, M. J., Little, J. P., van Essen, M., Wilkin, G. P., Burgomaster, K. A., Safdar, A., et al. (2006). Short-term sprint interval versus traditional endurance training: similar initial adaptations in human skeletal muscle and exercise performance. J. Physiol. 575(Pt 3), 901-911. doi: 10.1113/jphysiol.2006. 112094

Gibala, M. J., and McGee, S. L. (2008). Metabolic adaptations to short-term highintensity interval training: a little pain for a lot of gain? Exerc. Sport Sci. Rev. 36, 58-63. doi: 10.1097/JES.0b013e318168ec1f

Gillen, J. B., and Gibala, M. J. (2013). Is high-intensity interval training a timeefficient exercise strategy to improve health and fitness? Appl. Physiol. Nutr. Metab. 39, 409-412. doi: 10.1139/apnm-2013-0187

Gomez-Pinilla, F., Vaynman, S., and Ying, Z. (2008). Brain-derived neurotrophic factor functions as a metabotrophin to mediate the effects of exercise on cognition. Eur. J. Neurosci. 28, 2278-2287. doi: 10.1111/j.1460-9568.2008. 06524.x

Hanyu, O., Yamatani, K., Ikarashi, T., Soda, S., Maruyama, S., Kamimura, T., et al. (2003). Brain-derived neurotrophic factor modulates glucagon secretion from pancreatic alpha cells: its contribution to glucose metabolism. Diabetes. Obes. Metab 5, 27-37. doi: 10.1046/j.1463-1326.2003.00238.x

Heisz, J. J., Tejada, M. G., Paolucci, E. M., and Muir, C. (2016). Enjoyment for high-intensity interval exercise increases during the first six weeks of training: implications for promoting exercise adherence in sedentary adults. PLoS One 11:e0168534. doi: 10.1371/journal.pone.0168534

Iaia, F. M., Fiorenza, M., Perri, E., Alberti, G., Millet, G. P., and Bangsbo, J. (2015). The effect of two speed endurance training regimes on performance of soccer players. PLoS One 10:e0138096. doi: 10.1371/journal.pone.0138096

Jiménez-Maldonado, A., de Álvarez-Buylla, E. R., Montero, S., Melnikov, V., Castro-Rodríguez, E., Gamboa-Domínguez, A., et al. (2014). Chronic exercise increases plasma brain-derived neurotrophic factor levels, pancreatic islet size, and insulin tolerance in a TrkB-dependent manner. PLoS One 9:e115177. doi: 10.1371/journal.pone.0115177

Kahlert, S., Zundorf, G., and Reiser, G. (2005). Glutamate-mediated influx of extracellular $\mathrm{Ca} 2+$ is coupled with reactive oxygen species generation in cultured hippocampal neurons but not in astrocytes. J. Neurosci. Res. 79, 262-271. doi: $10.1002 /$ jnr.20322

Kang, J., and Ratamess, N. (2014). Which comes first? Resistance before aerobic exercise or vice versa? ACSM's Health Fit. J. 18, 9-14. doi: 10.1249/FIT. 0000000000000004

Krabbe, K. S., Nielsen, A. R., Krogh-Madsen, R., Plomgaard, P., Rasmussen, P., Erikstrup, C., et al. (2007). Brain-derived neurotrophic factor (BDNF) and type 2 diabetes. Diabetologia 50, 431-438. doi: 10.1007/s00125-0060537-4

Li, B., Lang, N., and Cheng, Z.-F. (2016). Serum levels of brain-derived neurotrophic factor are associated with diabetes risk, complications, and obesity: a cohort study from chinese patients with type 2 diabetes. Mol. Neurobiol. 53, 5492-5499. doi: 10.1007/s12035-015-9461-2

Lira, F. S., dos Santos, T., Caldeira, R. S., Inoue, D. S., Panissa, V. L. G., CabralSantos, C., et al. (2017). Short-term high- and moderate-intensity training 
modifies inflammatory and metabolic factors in response to acute exercise. Front. Physiol. 8:856. doi: 10.3389/fphys.2017.00856

Lommatzsch, M., Zingler, D., Schuhbaeck, K., Schloetcke, K., Zingler, C., SchuffWerner, P., et al. (2005). The impact of age, weight and gender on BDNF levels in human platelets and plasma. Neurobiol. Aging 26, 115-123. doi: 10.1016/j. neurobiolaging.2004.03.002

Lucas, S. J., Cotter, J. D., Brassard, P., and Bailey, D. M. (2015). High-intensity interval exercise and cerebrovascular health: curiosity, cause, and consequence. J. Cereb. Blood Flow Metab. 35, 902-911. doi: 10.1038/jcbfm.2015.49

Machado, A. F., Baker, J. S., Figueira Junior, A. J., and Bocalini, D. S. (2017). High-intensity interval training using whole-body exercises: training recommendations and methodological overview. Clin. Physiol. Funct. Imaging doi: 10.1111/cpf.12433 [Epub ahead of print].

Mador, M. J., Bozkanat, E., Aggarwal, A., Shaffer, M., and Kufel, T. J. (2004). Endurance and strength training in patients with COPD. Chest 125, 2036-2045. doi: 10.1378/chest.125.6.2036

Matthews, V. B., Åström, M.-B., Chan, M. H. S., Bruce, C. R., Krabbe, K. S., Prelovsek, O., et al. (2009). Brain-derived neurotrophic factor is produced by skeletal muscle cells in response to contraction and enhances fat oxidation via activation of AMP-activated protein kinase. Diabetologia 52, 1409-1418. doi: 10.1007/s00125-009-1364-1

Mowla, S. J., Farhadi, H. F., Pareek, S., Atwal, J. K., Morris, S. J., Seidah, N. G., et al. (2001). Biosynthesis and post-translational processing of the precursor to brain-derived neurotrophic factor. J. Biol. Chem. 276, 12660-12666. doi: 10.1074/jbc.M008104200

Murawska-Cialowicz, E., Wojna, J., and Zuwala-Jagiello, J. (2015). Crossfit training changes brain-derived neurotrophic factor and irisin levels at rest, after wingate and progressive tests, and improves aerobic capacity and body composition of young physically active men and women. J. Physiol. Pharmacol. 66, $811-821$.

Oliff, H. S., Berchtold, N. C., Isackson, P., and Cotman, C. W. (1998). Exerciseinduced regulation of brain-derived neurotrophic factor (BDNF) transcripts in the rat hippocampus. Brain Res. Mol. Brain Res. 61, 147-153. doi: 10.1016/ S0169-328X(98)00222-8

Physical Activity Guidelines Advisory Committee (2008). Physical Activity Guidelines Advisory Committee Report. Washington, DC: Physical Activity Guidelines Advisory Committee.

Poehlman, E. T., Dvorak, R. V., DeNino, W. F., Brochu, M., and Ades, P. A. (2000). Effects of resistance training and endurance training on insulin sensitivity in nonobese, young women: a controlled randomized trial. J. Clin. Endocrinol. Metab. 85, 2463-2468. doi: 10.1210/jcem.85.7.6692

Pugazhenthi, S., Nesterova, A., Jambal, P., Audesirk, G., Kern, M., Cabell, L., et al. (2003). Oxidative stress-mediated down-regulation of bcl-2 promoter in hippocampal neurons. J. Neurochem. 84, 982-996. doi: 10.1046/j.1471-4159. 2003.01606.x

Radak, Z., Suzuki, K., Higuchi, M., Balogh, L., Boldogh, I., and Koltai, E. (2016). Physical exercise, reactive oxygen species and neuroprotection. Free Radic. Biol. Med. 98, 187-196. doi: 10.1016/j.freeradbiomed.2016.01.024

Rasmussen, P., Brassard, P., Adser, H., Pedersen, M. V., Leick, L., Hart, E., et al. (2009). Evidence for a release of brain-derived neurotrophic factor from the brain during exercise. Exp. Physiol. 94, 1062-1069. doi: 10.1113/expphysiol. 2009.048512

Robinson, M. M., Lowe, V. J., and Nair, K. S. (2018). Increased brain glucose uptake after 12 weeks of aerobic high-intensity interval training in young and older adults. J. Clin. Endocrinol. Metab. 103, 221-227. doi: 10.1210/jc.201701571

Rudge, J. S., Mather, P. E., Pasnikowski, E. M., Cai, N., Corcoran, T., Acheson, A., et al. (1998). Endogenous BDNF protein is increased in adult rat hippocampus after a kainic acid induced excitotoxic insult but exogenous BDNF is not neuroprotective. Exp. Neurol. 149, 398-410. doi: 10.1006/exnr.1997. 6737

Saanijoki, T., Nummenmaa, L., Eskelinen, J.-J., Savolainen, A. M., Vahlberg, T., Kalliokoski, K. K., et al. (2015). Affective responses to repeated sessions of high-intensity interval training. Med. Sci. Sports Exerc. 47, 2604-2611. doi: 10.1249/mss.0000000000000721

Saanijoki, T., Nummenmaa, L., Koivumäki, M., Löyttyniemi, E., Kalliokoski, K. K., and Hannukainen, J. C. (2018). Affective adaptation to repeated SIT and MICT protocols in insulin-resistant subjects. Med. Sci. Sports Exerc. 50, 18-27. doi: 10.1249/mss.0000000000001415
Sanchez-Sanchez, J., Carretero, M., Ramirez-Campillo, R., Petisco, C., Diego, M., Gonzalo-Skok, O., et al. (2018). Effects of high-intensity training with one versus three changes of direction on youth female basketball players' performance. Kinesiology 50(Suppl. 1), 117-125.

Santos-Concejero, J., Billaut, F., Grobler, L., Olivan, J., Noakes, T. D., and Tucker, R. (2017). Brain oxygenation declines in elite Kenyan runners during a maximal interval training session. Eur. J. Appl. Physiol. 117, 1017-1024. doi: 10.1007/ s00421-017-3590-4

Satriotomo, I., Nichols, N. L., Dale, E. A., Emery, A. T., Dahlberg, J. M., and Mitchell, G. S. (2016). Repetitive acute intermittent hypoxia increases growth/neurotrophic factor expression in non-respiratory motor neurons. Neuroscience 322, 479-488. doi: 10.1016/j.neuroscience.2016.02.060

Saucedo Marquez, C. M., Vanaudenaerde, B., Troosters, T., and Wenderoth, N. (2015). High-intensity interval training evokes larger serum BDNF levels compared with intense continuous exercise. J. Appl. Physiol. 119, 1363-1373. doi: 10.1152/japplphysiol.00126.2015

Schleppenbach, L. N., Ezer, A. B., Gronemus, S. A., Widenski, K. R., Braun, S. I., and Janot, J. M. (2017). Speed- and circuit-based high-intensity interval training on recovery oxygen consumption. Int. J. Exerc. Sci. 10, 942-953.

Seifert, T., Brassard, P., Wissenberg, M., Rasmussen, P., Nordby, P., Stallknecht, B., et al. (2010). Endurance training enhances BDNF release from the human brain. Am. J. Physiol. Regul. Integr. Comp. Physiol. 298, R372-R377. doi: 10.1152/ ajpregu.00525.2009

Slusher, A. L., Patterson, V. T., Schwartz, C. S., and Acevedo, E. O. (2018). Impact of high intensity interval exercise on executive function and brain derived neurotrophic factor in healthy college aged males. Physiol. Behav. 191, 116-122. doi: 10.1016/j.physbeh.2018.04.018

So, J. H., Huang, C., Ge, M., Cai, G., Zhang, L., Lu, Y., et al. (2017). Intense exercise promotes adult hippocampal neurogenesis but not spatial discrimination. Front. Cell. Neurosci. 11:13. doi: 10.3389/fncel.2017. 00013

Stöggl, T. L., and Björklund, G. (2017). High intensity interval training leads to greater improvements in acute heart rate recovery and anaerobic power as high volume low intensity training. Front. Physiol. 8:562. doi: 10.3389/fphys.2017. 00562

Takuma, K., Lee, E., Kidawara, M., Mori, K., Kimura, Y., Baba, A., et al. (1999). Apoptosis in $\mathrm{Ca} 2+$ reperfusion injury of cultured astrocytes: roles of reactive oxygen species and NF-kappaB activation. Eur. J. Neurosci. 11, 4204-4212. doi: 10.1046/j.1460-9568.1999.00850.x

Talanian, J. L., Galloway, S. D., Heigenhauser, G. J., Bonen, A., and Spriet, L. L. (2007). Two weeks of high-intensity aerobic interval training increases the capacity for fat oxidation during exercise in women. J. Appl. Physiol. 102, 1439-1447. doi: 10.1152/japplphysiol.01098. 2006

Tsuchida, A., Nakagawa, T., Itakura, Y., Ichihara, J., Ogawa, W., Kasuga, M., et al. (2001). The effects of brain-derived neurotrophic factor on insulin signal transduction in the liver of diabetic mice. Diabetologia 44, 555-566. doi: 10. 1007/s001250051661

Tyler, W. J., and Pozzo-Miller, L. D. (2001). BDNF enhances quantal neurotransmitter release and increases the number of docked vesicles at the active zones of hippocampal excitatory synapses. J. Neurosci. 21, 4249-4258. doi: 10.1523/JNEUROSCI.21-12-04249.2001

Vaynman, S., Ying, Z., and Gomez-Pinilla, F. (2003). Interplay between brainderived neurotrophic factor and signal transduction modulators in the regulation of the effects of exercise on synaptic-plasticity. Neuroscience 122, 647-657. doi: 10.1016/j.neuroscience.2003.08.001

Vaynman, S., Ying, Z., and Gomez-Pinilla, F. (2004). Exercise induces BDNF and synapsin I to specific hippocampal subfields. J. Neurosci. Res. 76, 356-362. doi: 10.1002/jnr.20077

Wang, H., Yuan, G., Prabhakar, N. R., Boswell, M., and Katz, D. M. (2006). Secretion of brain-derived neurotrophic factor from PC12 cells in response to oxidative stress requires autocrine dopamine signaling. J. Neurochem. 96, 694-705. doi: 10.1111/j.1471-4159.2005.03572.x

Weston, K. S., Wisløff, U., and Coombes, J. S. (2014). High-intensity interval training in patients with lifestyle-induced cardiometabolic disease: a systematic review and meta-analysis. Br. J. Sports Med. 48, 1227-1234. doi: 10.1136/ bjsports-2013-092576

Yamanaka, M., Itakura, Y., Inoue, T., Tsuchida, A., Nakagawa, T., Noguchi, H., et al. (2006). Protective effect of brain-derived neurotrophic factor on pancreatic 
islets in obese diabetic mice. Metab. Clin. Exp. 55, 1286-1292. doi: 10.1016/j. metabol.2006.04.017

Yang, J., Ruchti, E., Petit, J. M., Jourdain, P., Grenningloh, G., Allaman, I., et al. (2014). Lactate promotes plasticity gene expression by potentiating NMDA signaling in neurons. Proc. Natl. Acad. Sci. U.S.A. 111, 12228-12233. doi: 10. 1073/pnas.1322912111

Yang, J. L., Lin, Y. T., Chuang, P. C., Bohr, V. A., and Mattson, M. P. (2014). BDNF and exercise enhance neuronal DNA repair by stimulating CREB-mediated production of apurinic/apyrimidinic endonuclease 1. Neuromol. Med. 16, 161-174. doi: 10.1007/s12017-013$8270-\mathrm{x}$
Conflict of Interest Statement: The authors declare that the research was conducted in the absence of any commercial or financial relationships that could be construed as a potential conflict of interest.

Copyright @ 2018 Jiménez-Maldonado, Rentería, García-Suárez, Moncada-Jiménez and Freire-Royes. This is an open-access article distributed under the terms of the Creative Commons Attribution License (CC BY). The use, distribution or reproduction in other forums is permitted, provided the original author(s) and the copyright owner(s) are credited and that the original publication in this journal is cited, in accordance with accepted academic practice. No use, distribution or reproduction is permitted which does not comply with these terms. 